DOI: $10.35643 /$ Info.25.2.2

Artículo original

\title{
Preservar la producción académica digital para el futuro: políticas diseñadas en los repositorios de Argentina ${ }^{1}$
}

\author{
Preserving academic production for the future: digital preservation policies \\ in national repositories of Argentina \\ Preservando a produção acadêmica digital para o futuro: políticas \\ desenhadas nos repositórios da Argentina
}

\section{María Cecilia Corda ${ }^{a}$ \\ Mariela Viñas ${ }^{\mathrm{b}}$}

Camila Vallefín ${ }^{c}$

\footnotetext{
a Instituto de Investigaciones Sociales de América Latina (FLACSO-CONICET, Argentina); Instituto de Investigaciones en Humanidades y Ciencias Sociales (Facultad de Humanidades y Ciencias de la Educación, Universidad Nacional de La Plata, La Plata, Argentina). Correo electrónico: mcorda@fahce.unlp.edu.ar. ORCID: 0000-0003-1885-7785.

${ }^{\mathrm{b}}$ Instituto de Investigaciones en Humanidades y Ciencias Sociales (Facultad de Humanidades y Ciencias de la Educación, Universidad Nacional de La Plata, La Plata, Argentina). Correo electrónico: mvinas@fahce.unlp.edu.ar. ORCID: 0000-0002-4243-1445.

${ }^{c}$ Instituto de Investigaciones en Humanidades y Ciencias Sociales (Facultad de Humanidades y Ciencias de la Educación, Universidad Nacional de La Plata, La Plata, Argentina). Correo electrónico: cvallefin@fahce.unlp.edu.ar. ORCID: 0000-0002-6872-2339.
}

\section{Resumen}

Dentro de la preservación digital se engloban todas aquellas acciones necesarias para poder reproducir un documento digital fiel al original y garantizar su acceso y accesibilidad, más allá del paso del tiempo y de los avances o cambios que se vayan sucediendo en la tecnología. Las técnicas usuales para llevar a cabo este proceso requieren de un esfuerzo constante, así como de la planificación a corto, mediano y largo plazo. De esta manera, la elaboración de políticas que incluyan la renovación de la información grabada y la actualización de los diversos formatos de datos es lo que debería garantizar la vida útil de los recursos digitales. El presente trabajo analiza entonces, mediante el análisis documental, las políticas vigentes acerca de la preservación digital en los 42 repositorios institucionales argentinos presentes en el Sistema Nacional de Repositorios Digitales (SNRD), dependiente del Ministerio de Ciencia, Tecnología e Innovación Productiva (MINCYT). A través del relevamiento de sus políticas de resguardo de la información, disponibles en sus sitios web, se valora la percepción e importancia que le brindan estas instituciones a la preservación de documentos académicos y 
cuáles son las estrategias o acciones manifiestas en ellas para minimizar la pérdida de información.

Palabras clave: PRESERVACIÓN DIGITAL; REPOSITORIOS; RECURSOS DIGITALES; POLÍTICAS DE PRESERVACIÓN; ARGENTINA.

\begin{abstract}
Digital preservation includes all fundamental actions to reproduce a digital document in a faithful way and to guarantee its accessibility beyond time and technology advances. The usual techniques to carry out this process require constant effort, as well as short, medium and long-term planning. In this way, the elaboration of policies that include the renewal of the recorded information and the updating of the data formats is what should guarantee the useful life of digital resources. This work examine, through the documentary analysis technique, the current policies of digital preservation in the 42 Argentine institutional repositories contained on the National System of Digital Repositories (SNRD), under the Ministry of Science, Technology and Productive Innovation (MINCYT). Through the survey of their own information protection policies, available on their websites, we will show the perception and value that the institutions give to the preservation of their academic documents and the strategies or actions manifested in them to minimize the loss of information.
\end{abstract}

Keywords: DIGITAL PRESERVATION; REPOSITORIES; DIGITAL RESOURCES; PRESERVATION POLICIES; ARGENTINA.

\title{
Resumo
}

A preservação digital engloba todas as ações necessárias para poder reproduzir um documento digital fiel ao original e garantir sua acessibilidade e acessibilidade, além da passagem do tempo e dos avanços ou mudanças que ocorrem na tecnologia. As técnicas usuais para a execução deste processo requerem esforço constante, bem como planejamento a curto, médio e longo prazo. Desta forma, a elaboração de políticas que incluam a renovação das informações registradas e a atualização dos diversos formatos de dados é o que deve garantir a vida útil dos recursos digitais. O presente trabalho analisa então, por meio de análise documental, as políticas atuais de preservação digital nos 42 repositórios institucionais argentinos presentes no Sistema Nacional de Repositórios Digitais (SNRD), dependentes do Ministério de Ciência, Tecnologia e Inovação Produtiva (MINCYT). Por meio do levantamento de suas políticas de proteção de informações, disponibilizadas em seus sites, avalia-se a percepção e importância que essas instituições atribuem à preservação de documentos acadêmicos e quais as estratégias ou ações nelas manifestadas para minimizar perdas. de informação.

Palavras-chave: PRESERVAÇÃO DIGITAL; REPOSITÓRIOS; RECURSOS DIGITAIS; POLÍTICAS DE PRESERVAÇÃO; ARGENTINA.

Fecha de recibido: $\quad 30 / 06 / 2020$

Fecha de aceptado: 21/07/2020 


\section{Acerca de los problemas de la preservación digital}

Las tecnologías de la información han transformado sin dudas las maneras de producir, procesar, guardar, difundir, recuperar y acceder a la información, propiciando una diversificación y un incremento sustancial de la información bibliográfica disponible en el entorno web, que, junto con las posibilidades de comunicación que ofrece el universo de Internet, dejan al alcance una gran cantidad de recursos y servicios de información digital (Suárez Maresma, 2018). Más allá de lo positivo que a un golpe de vista pueda generar dicho desarrollo, este trae aparejadas también otras responsabilidades por quienes los gestionan (muchas veces las mismas bibliotecas académicas), ya que requieren mecanismos para su procesamiento, almacenamiento y preservación en los espacios creados para ello, con el objetivo de que se pueda acceder en cualquier momento, y muchas veces desde cualquier lugar, a esas informaciones plasmadas en documentos de diversa índole.

Es preciso que los procesos antes mencionados sean realizados de tal manera que la información se pueda consultar tanto a corto plazo como también a través del tiempo. Esta posibilidad de acceso perdurable es necesaria porque la información podría ser indispensable para apoyar en tiempos futuros a la ciencia, la educación y la cultura, entre otros aspectos de la vida social. Preservar la información y el conocimiento a través del tiempo y el espacio es una preocupación de vieja data, que hoy en día se vuelve a ver desafiada e intenta resolverse a través de la elaboración de normas internacionales y nacionales, acumulación de experiencias en distintos ámbitos e investigación en la esfera académica.

En este sentido, ya desde mediados de los noventa han comenzado a desarrollarse diversos proyectos, investigaciones e incluso alternativas prácticas tendientes a lograr la preservación de los recursos digitales (Mourlot Rodríguez y Acosta Herrera, 2014). No obstante, según Suárez Maresma (2018), estas actividades, llevadas a cabo en muchos casos por las universidades, las bibliotecas o los archivos, se han desarrollado fundamentalmente en países centrales como Estados Unidos, Canadá, Australia, Nueva Zelanda, Gran Bretaña, Francia y Holanda, entre otros, y han sido acompañadas por pautas y recomendaciones ofrecidas por instituciones internacionales, entre las que se pueden mencionar a la International Federation of Library Associations and Institutions (IFLA), el International 
Council on Archives (ICA), la International Organization for Standardization (ISO), la Unesco, el Online Computer Library Center (OCL) y el Research Libraries Group (RLG).

Fundamentalmente, los problemas que giran en torno a la preservación digital se vinculan a los riesgos generados, por un lado, por degradación de datos, también conocida por «degradación»o «descomposición de bits»o «de datos» (o por su equivalente inglés de bit rot, que se refiere a una gradual degradación del medio de almacenaje o de los programas con el paso del tiempo). Por otro, por la obsolescencia tecnológica de formatos, soportes, hardware y software; por las fallas tecnológicas o los errores en la comunicación; por metadatos incompletos o ausencia de ellos; por ataques informáticos (comúnmente conocidos por el término en inglés: hackers); por errores humanos; por fenómenos o catástrofes socioambientales, entre otros riesgos posibles.

En el ámbito internacional, existe una batería de normas vinculadas con la problemática de la preservación de los objetos digitales, tales como la norma ISO 14.721-2012 (Organismo Internacional de Estandarización, 2012); la norma ISO 19.264-2017 (Organización Internacional de Estandarización, 2017), y la norma ISO 30.301-2019, adoptada recientemente en la Argentina por el IRAM (Instituto Argentino de Normalización y Certificación - Organización Internacional de Estandarización, 2019).

Desde hace más de dos décadas, en distintos países se han implementado y desarrollado repositorios institucionales (RI), dentro de la línea de la filosofía del acceso abierto ('AA', traducción de Open access, 'OA'). No alejada de esta realidad, la Argentina ha visto florecer, primero en el ámbito público, más tarde en el privado, una cantidad interesante de RI que propenden a recopilar, procesar, almacenar y difundir el conocimiento que se genera en el marco de las instituciones académicas.

Específicamente, la norma ISO 16.363-2017 (Una Norma Española - Organización Internacional de Estandarización, 2017) se refiere a los RI y sus aspectos de preservación digital a largo plazo.

La función principal de los RI es justamente la de garantizar la preservación de la producción, además de, por supuesto, el acopio, la organización, el acceso y la 
difusión de la producción académica, científica, administrativa o normativa de las instituciones, según sostiene Lynch (2003).

A esta conceptualización se puede añadir la que brinda más recientemente Ferreras Fernández, quien define a los RI como un conjunto de servicios que pueden prestar tanto las universidades como otros entes de investigación, dirigidos hacia la comunidad para «recopilar, administrar, difundir y preservar la producción documental digital generada en la institución, cualquiera sea su tipología, a través de la creación de una colección digital organizada, abierta e interoperable» (2018, p. 44), con el fin de aumentar la visibilidad y el impacto de estas informaciones.

Así, la preservación digital es una categoría inevitable en cualquier abordaje sobre los RI, independientemente del tipo de información que contengan o los procesos que se desarrollen en su seno. Estos deberían tener la capacidad de preservar sus recursos informacionales de manera segura y fidedigna a lo largo del tiempo.

\section{Sobre la preservación digital y su relación con los repositorios}

La bibliografía relacionada a la preservación cultural y digital, documentos digitales y RI es muy abundante e interesante con respecto a las investigaciones que se han llevado adelante, sobre todo en el ámbito bibliotecológico. Resultan valiosos los aportes conceptuales y sobre casos, así como las revisiones bibliográficas efectuadas por (en orden cronológico): Serra Serra (2001), Bia y Sánchez (2003), Candás (2006), Orera Orera (2008), Paradelo Luque (2009) y Térmens (2009); también las que han realizado más recientemente Díez Carrera (2012), Merlo Vega y Ferreras Fernández (2013), De Giusti (2014), Giordanino (2014), Ayala Salinas y Garcete (2015), Villanueva Rivas (2015), Álvarez Wong (2017), Corda, Viñas y Vallefín (2019), Ravelo Díaz, Mena Mugica y Del Castillo Guevara (2019), Suárez Quiceno y Osorio Tuberquia (2019).

Para Álvarez Wong (2017) la preservación digital implica acciones específicas, cuyo fin ulterior y a largo plazo es asegurar la permanencia y el acceso del contenido de documentos digitales a lo largo del tiempo y las tecnologías, independientemente del soporte, formato o sistema en que se encuentren.

Este autor sugiere ciertos principios de la preservación digital: 
1. Integridad (mantener las propiedades significativas del objeto, o sea, que la información no se altere).

2. Autenticidad (conservar su cualidad del original, acreditar su no alteración y pérdida de información, asegurando que lo que se preserva sea auténtico).

3. Fiabilidad (mantener la confianza en el contenido preservado).

4. Funcionalidad (que conserve las características mínimas para ser reproducido).

La preservación digital es uno de los procesos de gestión documental que supone tanto un conjunto de actividades archivísticas y tecnológicas como procesos estratégicos que tienen que ver con la planificación, la clasificación, la selección, el acceso a los materiales en sus distintos formatos, la descripción y la asignación de metadatos. Este complejo entramado muestra la necesidad de priorizar los niveles de ejecución e implementación de sistemas integrados de preservación por todas las entidades que tienen a su cargo la preservación de la información, fundamentalmente, los archivos y las bibliotecas (Sáenz Giraldo, 2019).

Por su parte, Carrazana Castro (2014) señala que la preservación digital refiere al conjunto de los procesos destinados a garantizar la continuidad de los objetos que constituyen el patrimonio documental digital durante todo el tiempo que se proyecte como necesario y útil. Y ante este desafío resalta que el principal impedimento de la preservación digital es la incompatibilidad de software y hardware que dio lugar a esos objetos digitales en el pasado y los que existen en la actualidad, o existirán en el futuro.

Asimismo, uno de los interrogantes más inquietantes que sobrevuela a las ciencias de la información es cómo mantener los objetos digitales y preservar su contenido informativo durante su ciclo de vida (Ponjuán Dante, 2005), dado que la veracidad e integridad de los documentos constituyen un aspecto medular, principalmente si se habla de generación de conocimiento como la que se da en el ámbito de universidades, centros e institutos de investigación.

En sí, la preservación digital se refiere a los procesos destinados a garantizar el acceso y la accesibilidad permanentes de los objetos digitales ${ }^{2}$. A tal fin, es necesario que la comprensión y la gestión de los objetos digitales se realice considerándolos desde cuatro puntos de vista: como fenómenos físicos, como 
codificaciones lógicas, como objetos conceptuales comprensibles para el ser humano y como conjuntos de elementos esenciales que deben ser preservados para ofrecer lo esencial del objeto a quienes lo usarán en un futuro (Unesco, 2003).

Se trata de un gran desafío para los archivos y las bibliotecas llevar a adelante las tareas de realizar copias de seguridad (backups) en soportes duraderos; efectuar sus renovaciones haciendo a su vez copias fidedignas del original; insertar metadatos básicos para recuperar la información y desplegarla visual y adecuadamente; migrar periódicamente entre configuraciones de tecnologías de la información en diferentes formatos; conservar estas tecnologías y sus sistemas o actualizar las versiones de software; y, por último, propiciar la arqueología digital, recuperando la información a partir de fuentes de datos que puedan estar dañadas, fragmentadas o resulten arcaicas. Precisamente, las políticas de los RI deben centrarse en la preservación a largo plazo de los objetos digitales que resulten íntegros y accesibles, y han de garantizar su preservación y acceso en el tiempo (Díez Carrera, 2012).

Para cumplir con este propósito, los RI deben expresar en las políticas su propósito a futuro. Estas, según Swan (2017), sirven para promover las intenciones y los objetivos del AA, crear interés y acciones, así como servir de ejemplo a otras instituciones.

Un trabajo interesante es el que realizaron los ya mencionados Ravelo Díaz, Mena Mugica y Del Castillo Guevara (2019), quienes estructuraron una propuesta de requisitos para la valoración de riesgos de preservación en RI, tomando en consideración el marco brindado por la norma internacional ISO 31.000. En el contexto de las políticas que las instituciones debieran darse, mencionan los siguientes puntos: establecer los objetivos, las metas, la visión y la misión del RI; identificar y designar su comunidad de personas usuarias, recursos y productores; apoyar financieramente al RI; conocer el marco regulatorio y los requerimientos legales; determinar el personal requerido; definir con verdadera precisión los procesos y sus respectivas acciones; implementar estrategias de preservación; establecer las estrategias de identificación y gestión de riesgos; realizar auditorías frecuentes a la organización y al sistema; proveer el acceso transparente a sus recursos y su documentación normativa. 
De este modo, ante los señalamientos de la bibliografía y la normativa internacional y nacional, así como la experiencia desarrollada por los RI en las últimas dos décadas, se ha planteado como propósito de la presente contribución el de presentar un análisis crítico de las políticas y sus referencias a la preservación digital en el marco de los RI incluidos en el SNRD. Este depende del Ministerio de Ciencia, Tecnología e Innovación Productiva de la República Argentina (MINCYT). Dicho sistema tiene como propósito conformar una red interoperable de RI en ciencia y tecnología, a partir del establecimiento de políticas, estándares y protocolos comunes a todos sus integrantes, generalmente situados en universidades nacionales y privadas, así como en institutos y centros de investigación.

\section{Metodología para el relevamiento y análisis de las políticas de preservación en los repositorios argentinos}

El abordaje realizado se basa en un análisis documental con el fin de relevar las distintas políticas disponibles en los sitios web de los 42 RI que se encuentran dentro del mencionado SNRD, los cuales se detallan en el anexo 1. Cabe mencionar que no son todos los que existen en el país, sino los que se enmarcan en el sistema citado al cumplir con los parámetros y requerimientos solicitados por el MINCYT.

Se efectuó un análisis documental en profundidad en torno a normas y políticas institucionales disponibles en los respectivos sitios web, para poder analizar aquellos aspectos relacionados con la preservación digital. Esto permitió trazar un panorama sobre las prácticas relativas a la cuestión en los RI y así poder efectuar una valoración acerca de la previsión de estas instituciones ante los riesgos digitales, al menos en lo que respecta a la información institucional que proveen de forma pública. Las acciones metodológicas se dividieron en:

- observación directa de los sitios web de los RI y recolección de políticas, normativas o documentos institucionales;

- análisis de contenido de la información recabada en esos espacios;

- recuperación de datos conceptuales y normativos específicos sobre la preservación digital en las políticas analizadas.

Este estudio se realizó entre los meses de marzo y mayo del año 2020. 


\section{Aspectos de preservación digital plasmados en las políticas de los repositorios nacionales}

En cuanto a la disponibilidad de las políticas en los RI, se encontraron 14 casos (33\%) que presentan alguna información en sus sitios web sobre su política institucional, contra 28 (67\%) que no la poseen. No obstante, la limitación de este análisis es que no resulta posible conocer si las instituciones (a) se encuentran en proceso de creación de sus políticas, con la intención de incorporar finalmente una declaración escrita de su compromiso sobre actividades de preservación digital o los riesgos digitales; (b) no tienen intención o recursos para confeccionar este tipo de documento, o (c) poseen políticas, pero no las disponen en AA.

Dentro de los RI suelen presentarse con el nombre de políticas, políticas y normativas y políticas, normativas y reglamentaciones.

Estos documentos suelen plasmar información relacionada con las características generales del RI, dónde se encuentran enmarcados (por ejemplo: universidades, facultades, institutos, centros, etc.), sus objetivos (generales y específicos), entre otras cuestiones. En varios de los casos estudiados se observa que el objetivo se centra en la reunión, el registro, la difusión y la preservación de la producción académica, científica e institucional de la entidad.

A su vez, en algunos RI se mencionan cuáles son los archivos que se pueden depositar en ellos, detallando los formatos adecuados, que suelen ser en .pdf (el más recomendado por todos; en algunos casos el material enviado en otros formatos es convertido a este), epub, .doc, .jpg, .tif, gif., ppt, .xlsx, entre otros, así como también para documentos audiovisuales en formato MPEG o AVI. Los idiomas aceptados del material suelen ser varios, no hay discriminación a la hora de su procesamiento en el ri. En otros, esta información se encuentra dentro del sitio principal o en el apartado de «Quiénes somos».

Los tipos de materiales que se admiten se encuentran divididos en libros o partes de libros, artículos, tesis de grado, tesis de posgrado, trabajos presentados en eventos (congresos, jornadas, etc.), planes de estudio, programas de asignaturas de grado y posgrado, proyectos e informes de investigación, pósteres de eventos, proyectos e informes de extensión, convenios, resúmenes de publicaciones, materiales de clases, reglamentaciones, fotografías, videos, etc. 
En las políticas se suele mencionar información relacionada con los alcances de la ley 26899 de Creación de Repositorios Digitales Institucionales de Acceso Abierto en Argentina, promulgada el 13 de noviembre del 2013 y reglamentada en diciembre de ese mismo año. En ese marco se brindan explicaciones sobre el alcance del AA y su desarrollo, el uso de las licencias Creative Commons (CC), se muestran resoluciones con relación a la creación de los RI en los marcos institucionales en que se desarrollan, entre otros tópicos.

En el cuadro 1 se describen las principales políticas que se plasman en los RI analizados. Estas responden a las que se hallaron en un mismo documento que se expone como política del recurso, o las que conforman párrafos en distintas partes del sitio web correspondiente.

\section{Cuadro 1: Tipos de políticas presentes en los repositorios argentinos}

\begin{tabular}{|c|c|}
\hline $\begin{array}{l}\text { Política } \\
\text { de } \\
\text { contenidos }\end{array}$ & $\begin{array}{l}\text { Relacionada con los materiales a incorporar en el RI. Entre otros temas, } \\
\text { incluye aspectos vinculados con el proceso de revisión; el de edición; la } \\
\text { publicación en el ámbito de la institución; la producción que realiza el } \\
\text { personal de la institución (equipo docente, de investigación, administrativo, } \\
\text { técnico y profesional) o centros asociados, y la puesta en valor de material } \\
\text { histórico-cultural que amerite su preservación. Asimismo, se suele detallar la } \\
\text { tipología de materiales aceptados, los idiomas y los formatos. }\end{array}$ \\
\hline $\begin{array}{l}\text { Política } \\
\text { de } \\
\text { acceso } \\
\text { de } \\
\text { datos }\end{array}$ & $\begin{array}{l}\text { Vinculada con la visualización y descarga de los materiales de forma libre y } \\
\text { gratuita, sin necesidad de registro o autorización previa. } \\
\text { También dentro de esta política se mencionan las licencias CC y temas de } \\
\text { propiedad intelectual contempladas. }\end{array}$ \\
\hline $\begin{array}{l}\text { Política } \\
\text { de } \\
\text { depósito }\end{array}$ & $\begin{array}{l}\text { Relacionada con las vías de depósito, tales como el autoarchivo, el depósito } \\
\text { delegado o el depósito designado con permiso especial a un/a representante } \\
\text { de una dependencia de la institución que asume el compromiso de } \\
\text { procesamiento de los materiales. }\end{array}$ \\
\hline $\begin{array}{l}\text { Política } \\
\text { de } \\
\text { metadatos }\end{array}$ & $\begin{array}{l}\text { Referida a los metadatos que se aplican sobre un recurso para facilitar su } \\
\text { organización, recuperación, preservación e interoperabilidad. Los RI emplean } \\
\text { por lo general el esquema sencillo de metadatos propuesto por Dublin Core. }\end{array}$ \\
\hline $\begin{array}{l}\text { Política } \\
\text { de digitalización }\end{array}$ & $\begin{array}{l}\text { Orientada a los criterios bajo los cuales se selecciona, prepara y digitaliza } \\
\text { material para ingresarlo en AA a través del RI. }\end{array}$ \\
\hline $\begin{array}{l}\text { Política de } \\
\text { servicios (para } \\
\text { personas } \\
\text { usuarias, autoras } \\
\text { y autores, otros } \\
\quad \text { RI) }\end{array}$ & $\begin{array}{l}\text { Enfocada en los servicios para personas usuarias (por ejemplo: instructivos } \\
\text { sobre cómo buscar material, acerca de la difusión, suscripciones, guías, } \\
\text { estadísticas, rankings, etc.); para autoras y autores (instructivos para efectuar } \\
\text { el autoarchivo, manuales de procedimientos, guías, talleres de capacitación o } \\
\text { actualización en relación a RI, AA, etc.); servicios para otros RI (por ejemplo: } \\
\text { cursos de capacitación, conferencias, manuales, exportación/importación de } \\
\text { registros, etc.). }\end{array}$ \\
\hline
\end{tabular}




\begin{tabular}{|c|l|}
\hline $\begin{array}{c}\text { Política } \\
\text { de } \\
\text { calidad }\end{array}$ & $\begin{array}{l}\text { Centrada en la aplicación de normas de calidad, tales como la ISO 9.001- } \\
\text { 2015, a partir de la cual se debe incluir un compromiso de mejorar } \\
\text { continuamente el sistema de gestión de calidad del RI. }\end{array}$ \\
\hline $\begin{array}{c}\text { Política de } \\
\text { preservación } \\
\text { digital }\end{array}$ & $\begin{array}{l}\text { Comprometida con el aseguramiento de ofrecer acceso, accesibilidad, } \\
\text { legibilidad e integridad de todas las obras que el RI alberga a tiempo } \\
\text { indefinido. }\end{array}$ \\
\hline
\end{tabular}

Fuente: elaboración propia

Los nombres con los que refieren a estas políticas en los RI son los siguientes: política de acceso y de uso a contenidos, política de accesibilidad y preservación, políticas de preservación, política de normalización de metadatos, políticas de uso de contenidos y políticas de gestión de derechos de autor.

En lo que respecta específicamente al tema de preservación digital, se percibe que solo algunos RI la abordan explícitamente. En algunas políticas sí se encuentra mencionada, pero en otras este aspecto solo se comenta de modo sumario en un apartado.

En ese universo de 14 RI, se describen sucintamente cuáles son dichas políticas, generalmente vinculadas a backups periódicos en servidores externos; vigilancia y conversión de formatos de archivo; actualización del software que sustenta al repositorio (los más empleados son DSpace y Greenstone); comprobaciones periódicas de la integridad de los archivos para asegurar que la obra no ha sufrido alteraciones; comprobaciones periódicas de la accesibilidad de la obra (verificación de privacidad de obras embargadas); inclusión de una URL persistente para la identificación y localización inequívoca de la obra; asignación y revisión de metadatos; entre otras acciones.

En cuatro de los RI se nombra de manera directa la cuestión de la preservación digital en los objetivos de la política. Es el caso de la Biblioteca Digital FCENUBA, perteneciente a la Facultad de Ciencias Exactas y Naturales de la Universidad de Buenos Aires, en donde se menciona que tiene por propósito «crear un archivo digital abierto de todos los materiales creados por la Facultad y sus miembros, en el curso de su actividad docente, investigadora y de gestión, con fines de preservación, acceso y difusión para toda la comunidad».

También el CEFA digital, del Centro Educativo de las Fuerzas Armadas, manifiesta el concepto entre sus objetivos: «Tiene como propósito fundamental asegurar la 
accesibilidad, legibilidad y preservación digital a tiempo indefinido de todas las obras que alberga».

En el Repositorio Institucional del Instituto Universitario de Ciencias de la Salud Fundación H. A. Barceló se plantea como objetivo principal el «asegurar la accesibilidad, legibilidad y preservación digital de todos los documentos allí alojados».

En esta misma línea se suma lo expresado por el SEDICI-UNLP, el cual manifiesta que su propósito fundamental es «asegurar la accesibilidad, legibilidad y preservación digital a tiempo indefinido de todas las obras que alberga».

Por su parte, en otros cuatro de los RI se plasma que, en caso de remover documentos, estos no serán eliminados físicamente, sino solamente de la consulta pública. En Producción académica de la Universidad Católica de Córdoba, además de tratarse de una medida que proceda por pedido expreso del autor o la autora, se detalla que la institución puede remover documentos sin su autorización en caso de que no sean pertinentes, los soportes o formatos no sean satisfactorios, contengan virus informáticos u otro problema técnico, sospechas de plagio o duplicados. Mientras tanto, en Memoria Académica de la Facultad de Humanidades y Ciencias de la Educación de la Universidad Nacional de La Plata se explicita que no se permitirán modificaciones en los documentos depositados, aunque sí listas de erratas y correcciones junto al registro original o una versión actualizada del documento. $\mathrm{Y}$ en el RDyD de la Universidad Nacional de Avellaneda se especifica que no se permitirán cambios en las obras depositadas y que se podrán depositar versiones actualizadas de una obra ya existente. En estas situaciones, habrá enlaces entre la versión original y las posteriores, con la versión más reciente identificada con claridad. Aclara que a pedido de los autores y las autoras la versión anterior podrá ser retirada de la visibilidad pública y que la URL persistente de la obra siempre apuntará a la última versión del objeto digital en cuestión. Por último, en el REDI (repositorio digital) de la Universidad FASTA se pauta que los documentos podrán ser eliminados por solicitud de autoras/es o propietarios/as de los derechos de autor debido a los siguientes motivos: la violación comprobada del derecho de autor, requerimientos legales y violaciones probadas, o investigaciones fraudulentas. También explica que los ítems retirados no son removidos del repositorio, pero sí de la vista pública; que no se permitirán 
cambios en los documentos depositados, y que, en caso de una actualización de un documento, esta puede ser depositada como un agregado, eliminando las versiones anteriores de la vista del público, pero no de la base de datos.

En el caso de CIC Digital se hace expresa la adhesión a las directrices de Unesco (2003), la norma OAIS y otras iniciativas internacionales que dan marco a la preservación digital.

En la terminología empleada, como ya se aclaró antes, suelen usarse como equivalentes los términos acceso y accesibilidad, sin tener en cuenta que este último posee un sentido específico.

Ninguna de las políticas incluye todos los componentes propuestos en el trabajo de Mourlot Rodríguez y Acosta Herrera (2014), referidos a alcance y contenido; propósito; mandato; objetivo de las actividades de preservación digital; retos de la preservación; políticas; principios generales; selección, valoración y adquisición; naturaleza de las colecciones: modelos de preservación - estrategia; almacenamiento, duplicación y respaldos; derechos y restricciones; criterios de acceso y uso; planificación financiera; metadatos y documentación; formatos y extensiones de los documentos; funciones y responsabilidades; colaboración, y, como aspecto adicional, glosario. A ellos se podría añadir lo remarcado por Ravelo Díaz, Mena Mugica y Del Castillo Guevara (2019) sobre establecer las estrategias de identificación y gestión de riesgos.

\section{Consideraciones finales}

Si bien es cierto que los RI representan una opción de AA para acceder a las publicaciones académicas, el poder hacerlo a lo largo del tiempo se torna una gran incertidumbre, coincidiendo en este punto con lo sostenido en el trabajo de Suárez Maresma (2018), por esto mismo, es fundamental el papel de quienes trabajan como profesionales de la información, teniendo en cuenta los aspectos primordiales de la preservación digital a largo plazo, preocupaciones que ya se han desarrollado en trabajos anteriores (Corda, Viñas, Vallefín, 2019).

Como resultado del análisis realizado en los 42 RI argentinos, se ha identificado que muchos se encuentran sin políticas explícitas sobre los tópicos de preservación del material que albergan, por lo menos de manera pública a través 
de sus sitios web. Y respecto al $33 \%$ que las posee, hay aspectos que requieren ser mayormente profundizados.

La preservación digital se constituye en un reto por brindar el acceso, la accesibilidad, la legibilidad y la autenticidad de los objetos digitales a través del tiempo, los dominios y los cambios del entorno técnico/tecnológico según las necesidades de las comunidades que vayan a usar la información digital. En el ámbito de las instituciones académicas es una necesidad la formulación y el diseño de políticas de preservación a largo plazo, sin pasar por alto en estas la importancia de conservar las cualidades de integridad, autenticidad, fiabilidad y funcionalidad de la documentación digital (Álvarez Wong, 2017).

Los problemas asociados con la dificultad de preservar estos aspectos de la información mantenida en los RI suponen diversos tipos de riesgos para las instituciones académicas, relacionados fundamentalmente con la tecnología, el tiempo, el personal y las dificultades económico-financieras para sostener y desarrollar las plataformas, por lo que la gestión de estos debería ser una prioridad para ellas.

Tal como Ravelo Díaz, Mena Mugica y Del Castillo Guevara (2019) indican, el cruce entre preservación digital y gestión del riesgo resulta particularmente esencial, ya que muchas veces el foco se pone en la selección, el análisis y el procesamiento de los objetos digitales en el RI, lo cual procura fomentar su crecimiento y desarrollo. En tanto, se descuidan otros procesos, como el que se analizó en el presente trabajo. Se pierde de vista la proyección del RI a futuro: ¿Cuáles son las acciones que la institución prevé para preservar los materiales? ¿Qué medidas se adaptan y adoptan para asegurar la perpetuidad de acceso y accesibilidad? ¿Qué rutinas en la gestión cotidiana se trazan para resguardar la información en los registros y objetos digitales en sí? ¿Cómo se planifica la actualización de las versiones de software y hardware? ¿Qué pérdidas está dispuesta a asumir la institución? Justamente uno de los puntos más importantes y difíciles en el campo de la preservación digital es seleccionar aquello que se debe conservar, asegurando su perpetuidad. En un contexto como el actual, en el que la sociedad depende en gran medida de los entornos digitales, el conocimiento que producen estas instituciones académicas no solo es necesario que sea seleccionado, analizado, procesado y almacenado, sino que es imprescindible que 
sea difundido, comunicado y adecuadamente preservado (Suárez Quiceno y Osorio Tuberquia, 2018).

Se tendrán que considerar seriamente los parámetros brindados por las normas internacionales, así como las pautas y experiencias en el ámbito internacional, regional y nacional a la hora de desarrollar las políticas en los RI.

En definitiva, y en consonancia con los recientes principios TRUST (Lin et al., 2020), los RI deben poder demostrar su transparencia, responsabilidad, foco en la comunidad usuaria, sostenibilidad y tecnología en pos de la preservación digital. Además, es esencial que se compartan en AA para que otras entidades, tales como universidades, institutos y centros de investigación, comiencen a transitar el camino de la explicitación de sus pautas (Swan, 2017), como una forma de saber cómo proceder hoy, así como también en el futuro, de la mano de quienes continúen la tarea de la comunicación académica y científica en el país.

\section{Referencias bibliográficas}

Álvarez Wong, B. I. (2017). Los repositorios digitales para la conservación: un acercamiento a la preservación digital a largo plazo. Ciencias de la Información, 48(2), 15-22. Recuperado de https://www.redalyc.org/pdf/1814/181454540003.pdf

Argentina. (2013, diciembre 9). Ley 26899: Repositorios digitales institucionales de acceso abierto. Recuperado de http://servicios.infoleg.gob.ar/infolegInternet/anexos/220000224999/223459/norma.htm

Ayala Salinas, M. E., y Garcete, W. (2015). Preservación y conservación de documentos bibliográficos en biblioteca universitaria. Revista Científica de la UCSA, 2(2). Recuperado de http://scielo.iics.una.py/pdf/ucsa/v2n2/v2n2a05.pdf

Bia, A., y Sánchez, M. (2003). Desarrollo de una política de preservación digital: tecnología, planificación y perseverancia. Recuperado de http://media.cervantesvirtual.com/research/articles/JBIDI02a.pdf

Candás, J. (2006). El papel de los metadatos en la preservación digital. El Profesional de la Información, 15(2), 126-136. Recuperado de http://eprints.rclis.org/8359/1/final.pdf 
Carrazana Castro, E. (2014). Retos y alternativas para la preservación a largo plazo de información digital en bibliotecas. Reflexiones, 10(10), 191-196. Recuperado de http://eprints.rclis.org/25238/1/15Reflexi\%C3\%B3n\%2003.pdf

Corda, M. C., Viñas, M., y Vallefín, C. (2019). Las bibliotecas universitarias frente a los desafíos, dilemas y riesgos de la preservación digital de los documentos académicos. X Jornadas de Temas Actuales en Bibliotecología, Mar del Plata, Argentina. Recuperado de http://www.memoria.fahce.unlp.edu.ar/trab_eventos/ev.13162/ev.13162.p $\underline{\mathrm{df}}$

De Giusti, M. R. (2014). Una metodología de evaluación de repositorios digitales para asegurar la preservación en el tiempo y el acceso a los contenidos. (Tesis de doctorado, Universidad Nacional de La Plata, La Plata).

Díez Carrera, C. (2012). La preservación digital en las bibliotecas nacionales de la Unión Europea: un análisis comparativo. ANABAD, 62(2). Recuperado de https://dialnet.unirioja.es/servlet/articulo?codigo $=4107462$

Ferreras Fernández, T. (2018). Los repositorios institucionales: evolución y situación actual en España. Recuperado de https://gredos.usal.es/handle/10366/138583

Giordanino, E. P. (2014). Preservación digital: buenas prácticas. Buenos Aires: Universidad de Buenos Aires. Recuperado de http://www.sisbi.uba.ar/sites/default/files/sisbi/presentaciones/Giordanino _2014_preservacion_bp_0.pdf

Instituto Argentino de Normalización y Certificación - Organización Internacional de Estandarización. (2015). Sistemas de gestión de la calidad: fundamentos y vocabulario (Norma IRAM-ISO n. ${ }^{\circ}$ 9001).

Instituto Argentino de Normalización y Certificación - Organización Internacional de Estandarización. (2019). Información y documentación - Sistemas de gestión para los documentos de la actividad. Requisitos (Norma iram-iso n. $\left.{ }^{\circ} 30.301\right)$.

Licencias. (s. f.). En Creative Commons Argentina. Recuperado de http://www.creativecommons.org.ar/licencias.html

Lin, D. et al. (2020). The TRUST principles for digital repositories (Trad. M. R. de Giusti). Scientific Data, 7, 144. doi:10.1038/s41597-020-0486-7 
Lynch, C.A. (2003). Institutional Repositories: Essential Infrastructure For Scholarship In The Digital Age. portal: Libraries and the Academy 3(2), 327-336. doi:10.1353/pla.2003.0039

Merlo Vega, J. A., y Ferreras Fernández, T. (2013). Preservación digital y difusión de la revista Educación y Biblioteca desde el repositorio Gredos de la Universidad de Salamanca. El Profesional de la Información, 22(2). Recuperado de http://eprints.rclis.org/18962/1/DBD_Merlo_ferreras_EducacionyBibliotec a.pdf

Mourlot Rodríguez, L. M., y Acosta Herrera, R. E. (2014). Política de preservación para el fondo digital de la biblioteca de la Casa de las Américas. Ciencias de la Información, 45(1). Recuperado de http://hdl.handle.net/20.500.11959/brapci/57850

Orera Orera, L. (2008). Preservación digital y bibliotecas: un nuevo escenario. Revista General de Información y Documentación, 18, 9-24. Recuperado de

https://revistas.ucm.es/index.php/RGID/article/view/RGID0808110009A/ $\underline{9220}$

Organización Internacional de Estandarización. (2009). Risk management: principles and guidelines (Norma iso n. ${ }^{\circ} 31.000$ ).

Organismo Internacional de Estandarización. (2012). Space data and information transfer systems - Open archival information system (oais) - Reference model (Norma iso n. ${ }^{\circ}$ 14.721).

Organización Internacional de Estandarización. (2017). Photography - Archiving systems - Image quality analysis - Part 1: Reflective originals (Norma iso n. $\left.{ }^{\circ} 19.264\right)$.

Paradelo Luque, A. M. (2009). Preservación documental en repositorios institucionales. Investigación Bibliotecológica: Archivonomía, Bibliotecología e Información, 23(49). Recuperado de http://www.scielo.org.mx/pdf/ib/v23n49/v23n49a9.pdf

Ponjuán Dante, G. (2005). Gestión de información: dimensiones e implementación para el éxito organizacional. Rosario: Nuevo Parhadigma.

Ravelo Díaz, G., Mena Mugica, M. M., y Del Castillo Guevara, J. (2019). Requisitos para la valoración de riesgos de preservación en repositorios digitales. Biblios: Journal of Librarianship and Information Science, 75, 
25-34. Recuperado de

https://biblios.pitt.edu/ojs/index.php/biblios/article/view/484/363

Sáenz Giraldo, A. (2019). La preservación digital en Colombia: un análisis desde la perspectiva normativa. Revista Interamericana de Bibliotecología, 42(1), 87-97. Recuperado de http://eprints.rclis.org/33913/1/v42n1a9.pdf

Serra Serra, J. (2001). Gestión de los documentos digitales: estrategias para su conservación. El Profesional de la Información, 10(9). Recuperado de http://www.elprofesionaldelainformacion.com/contenidos/2001/septiembr e/1.pdf

Suárez Maresma, I. (2018). Repositorios institucionales: una alternativa para la preservación digital. Congreso Internacional de Información. Info 2018: Información y conocimiento: desafíos para el desarrollo sostenible. La Habana, Cuba. Recuperado de http://www.congresoinfo.cu/index.php/info/info2018/paper/viewFile/735/440

Suárez Quiceno, C., y Osorio Tuberquia, A. (2019). Experiencias sobre preservación digital para bibliotecas y archivos: dos casos de estudio en Colombia. Revista Interamericana de Bibliotecología, 42(2). Recuperado de

https://aprendeenlinea.udea.edu.co/revistas/index.php/RIB/article/view/33 $\underline{5122}$

Swan, A. (2013). Directrices para políticas de desarrollo y promoción del acceso abierto. París: Unesco. Recuperado de https://unesdoc.unesco.org/ark:/48223/pf0000222536

Térmens, M. (2009). Investigación y desarrollo en preservación digital: un balance internacional. El Profesional de la Información, 18(6), 613-624. Recuperado de http://eprints.rclis.org/16218/1/Investigacion-preservacionEPI.pdf

Una Norma Española - Organización Internacional de Estandarización. (2017). Sistemas de transferencia de información y datos espaciales. Auditoría y certificación de repositorios digitales de confianza (Norma une-iso n. $^{\circ}$ 16.363).

Unesco (2003). Directrices para la preservación del patrimonio digital. París: Unesco. Recuperado de http://www.unesco.org/new/es/communicationand-information/resources/publications-and-communicationmaterials/publications/full-list/guidelines-for-the-preservation-of-digitalheritage/ 
Villanueva Rivas, D. (2015). Preservación digital de revistas en la unam y repositorios institucionales, un acercamiento. E-Ciencia de la Información, 5(1). Recuperado de https://dialnet.unirioja.es/descarga/articulo/5511048.pdf

\section{Notas}

1 Trabajo realizado en el marco del Proyecto UNLP «Innovación y riesgo en la gestión de bibliotecas de investigación en la Argentina», 2020-2022, dirigido por María Cecilia Corda.

${ }^{2}$ La accesibilidad web es el acceso universal a esta, independientemente del tipo de hardware, software, infraestructura de red, idioma, cultura, localización geográfica y capacidades de las personas usuarias (http://W3C.org). Es importante comprender la diferencia entre acceso y accesibilidad, ya que comúnmente se emplean de modo indistinto, pero su alcance conceptual es diferente.

Nota: El editor Mario Barité aprobó este artículo.

Nota de contribución: Corda 33\%, Viñas 33\% y Vallefin 34\%.

Corrección de estilo realizada por Vanina Castellano en el marco del convenio entre la Tecnicatura universitaria en corrección de estilo (FHCE) y la Facultad de Información y Comunicación (Udelar). 
Anexo 1

Repositorios del Sistema Nacional de Repositorios Digitales

\begin{tabular}{|c|c|c|}
\hline N. ${ }^{\mathbf{2}}$ & Nombre del RI & Sitio web \\
\hline 0 & $\begin{array}{l}\text { SNRD (Sistema Nacional de Repositorios } \\
\text { Digitales) }\end{array}$ & $\begin{array}{l}\text { https://repositoriosdigitales.mincyt.gob.ar/vufind/ } \\
\text { Content/repos }\end{array}$ \\
\hline 1 & $\begin{array}{l}\text { Argos, Universidad Nacional de Misiones, } \\
\text { Facultad de Humanidades y Ciencias } \\
\text { Sociales }\end{array}$ & http://argos.fhycs.unam.edu.ar/ \\
\hline 2 & $\begin{array}{l}\text { Biblioteca Digital, Universidad de Buenos } \\
\text { Aires, Facultad de Ciencias Económicas }\end{array}$ & http://bibliotecadigital.econ.uba.ar/ \\
\hline 3 & $\begin{array}{l}\text { **Biblioteca Digital FCEN-UBA, Facultad de } \\
\text { Ciencias Exactas y Naturales, Universidad } \\
\text { de Buenos Aires. }\end{array}$ & https://bibliotecadigital.exactas.uba.ar/ \\
\hline 4 & $\begin{array}{l}\text { Biblioteca Digital Uncuyo, Universidad } \\
\text { Nacional de Cuyo. }\end{array}$ & https://bdigital.uncu.edu.ar/ \\
\hline 5 & $\begin{array}{l}\text { Biblioteca Virtual, Universidad Nacional } \\
\text { del Litoral. }\end{array}$ & https://bibliotecavirtual.unl.edu.ar:8443/ \\
\hline 6 & $\begin{array}{l}\text { **CEFA digital, Centro Educativo de las } \\
\text { Fuerzas Armadas. }\end{array}$ & http://www.cefadigital.edu.ar/ \\
\hline 7 & $\begin{array}{l}\text { **CIC Digital, Repositorio Institucional } \\
\text { Comisión de Investigaciones Científicas. }\end{array}$ & https://digital.cic.gba.gob.ar/ \\
\hline 8 & $\begin{array}{l}\text { **Conicet Digital, Consejo Nacional de } \\
\text { Investigaciones Científicas y Tecnológicas. }\end{array}$ & https://ri.conicet.gov.ar/ \\
\hline 9 & $\begin{array}{l}\text { **Fauba Digital, Repositorio Institucional } \\
\text { Científico y Académico de la Facultad de } \\
\text { Agronomía, Universidad de Buenos Aires. }\end{array}$ & http://ri.agro.uba.ar/cgi-bin/library.cgi \\
\hline 10 & $\begin{array}{l}\text { Filo Digital UBA-FFYL, Facultad de Filosofía } \\
\text { y Letras, Universidad de Buenos Aires. }\end{array}$ & http://repositorio.filo.uba.ar/xmlui/ \\
\hline 11 & $\begin{array}{l}\text { INTA Digital, Instituto Nacional de } \\
\text { Tecnología Agropecuaria. }\end{array}$ & https://repositorio.inta.gob.ar/ \\
\hline 12 & $\begin{array}{l}\text { **Memoria Académica, Facultad de } \\
\text { Humanidades y Ciencias de la Educación, } \\
\text { Universidad Nacional de La Plata. }\end{array}$ & http://www.memoria.fahce.unlp.edu.ar/ \\
\hline 13 & $\begin{array}{l}\text { Naturalis, Facultad de Ciencias Naturales y } \\
\text { Museo, Universidad Nacional de La Plata. }\end{array}$ & http://naturalis.fenym.unlp.edu.ar/ \\
\hline 14 & $\begin{array}{l}\text { Nülan, Facultad de Ciencias Económicas y } \\
\text { Sociales, Universidad Nacional de Mar del } \\
\text { Plata. }\end{array}$ & http://nulan.mdp.edu.ar/ \\
\hline 15 & $\begin{array}{l}\text { **Producción académica, Universidad } \\
\text { Católica de Córdoba. }\end{array}$ & http://pa.bibdigital.uccor.edu.ar/ \\
\hline 16 & $\begin{array}{l}* * \text { RDYD, Universidad Nacional de } \\
\text { Avellaneda. }\end{array}$ & http://170.210.71.22/cgi-bin/library.cgi \\
\hline 17 & $* *$ REDI, Universidad fasta. & http://redi.ufasta.edu.ar:8080/xmlui/ \\
\hline 18 & REDIUNLu, Universidad Nacional de Luján. & https://ri.unlu.edu.ar/xmlui/ \\
\hline
\end{tabular}




\begin{tabular}{|c|c|c|}
\hline 19 & $\begin{array}{l}\text { RepHipUNR, Universidad Nacional de } \\
\text { Rosario. }\end{array}$ & https://rephip.unr.edu.ar/ \\
\hline 20 & $\begin{array}{l}\text { Repositorio Digital Biblioteca Central, } \\
\text { Universidad Nacional de Villa María. }\end{array}$ & $\begin{array}{l}\text { http://biblioteca.unvm.edu.ar/Repositorio/index.h } \\
\text { tml }\end{array}$ \\
\hline 21 & $\begin{array}{l}\text { **RDI UNC, Repositorio Digital } \\
\text { Institucional, Universidad Nacional del } \\
\text { Comahue. }\end{array}$ & http://rdi.uncoma.edu.ar/ \\
\hline 22 & $\begin{array}{l}\text { Repositorio Digital Institucional José María } \\
\text { Rosa, Universidad Nacional de Lanús. }\end{array}$ & $\begin{array}{l}\text { http://repositoriojmr.unla.edu.ar/greenstone/cgi- } \\
\text { bin/library.cgi }\end{array}$ \\
\hline 23 & $\begin{array}{l}\text { Repositorio Digital San Andrés, } \\
\text { Universidad de San Andrés. }\end{array}$ & http://repositorio.udesa.edu.ar/jspui/ \\
\hline 24 & $\begin{array}{l}\text { Repositorio Digital Universitario, } \\
\text { Universidad Nacional de Córdoba. }\end{array}$ & https://rdu.unc.edu.ar/ \\
\hline 25 & $\begin{array}{l}\text { **Repositorio Institucional (RI) del Instituto } \\
\text { Universitario de Ciencias de la Salud } \\
\text { Fundación H. A. Barceló. }\end{array}$ & $\begin{array}{l}\text { http://repositorio.barcelo.edu.ar/greenstone/cgi- } \\
\text { bin/library.cgi? } a=p \& p=\text { home\&l=es \&w=utf- } 8\end{array}$ \\
\hline 26 & $\begin{array}{l}\text { Repositorio Institucional Ucasal, } \\
\text { Universidad Católica de Salta. }\end{array}$ & $\begin{array}{l}\text { http://bibliotecas.ucasal.edu.ar/opac_css/index.ph } \\
\text { p?lvl=cmspage\&pageid=16 }\end{array}$ \\
\hline 27 & Riunne, Universidad Nacional del Nordeste. & http://repositorio.unne.edu.ar/ \\
\hline 28 & $\begin{array}{l}\text { Repositorio Institucional Digital de la UNA, } \\
\text { Universidad Nacional de las Artes. }\end{array}$ & http://repositorio.una.edu.ar/ \\
\hline 29 & $\begin{array}{l}\text { Repositorio Institucional Segemar (Servicio } \\
\text { Geológico Minero Argentino) }\end{array}$ & https://repositorio.segemar.gov.ar/ \\
\hline 30 & $\begin{array}{l}\text { **Repositorio RID-UNRN, Universidad } \\
\text { Nacional de Río Negro. }\end{array}$ & https://rid.unrn.edu.ar/jspui/ \\
\hline 31 & $\begin{array}{l}\text { Ridaa Unicen (Repositorio Institucional } \\
\text { Digital de Acceso Abierto), Universidad } \\
\text { Nacional del Centro de la Provincia de } \\
\text { Buenos Aires. }\end{array}$ & https://www.ridaa.unicen.edu.ar/xmlui/ \\
\hline 32 & $\begin{array}{l}\text { RIDAA-UNQ (Repositorio Digital de Acceso } \\
\text { Abierto - Universidad Nacional de Quilmes) }\end{array}$ & https://ridaa.unq.edu.ar/?locale-attribute $=$ en \\
\hline 33 & $\begin{array}{l}\text { Repositorio Institucional del Departamento } \\
\text { de Humanidades. Universidad Nacional del } \\
\text { Sur. }\end{array}$ & $\begin{array}{l}\text { http://repositoriodigital.uns.edu.ar/handle/123456 } \\
\underline{789 / 9}\end{array}$ \\
\hline 34 & $\begin{array}{l}\text { RPsico (Repositorio de la Facultad de } \\
\text { Psicología), Universidad Nacional de Mar } \\
\text { del Plata. }\end{array}$ & http://rpsico.mdp.edu.ar/ \\
\hline 35 & **Sedici, Universidad Nacional de La Plata. & http://sedici.unlp.edu.ar/ \\
\hline 36 & $\begin{array}{l}\text { Sistema de Gestión del Conocimiento ANLIS } \\
\text { Malbrán (Administración Nacional de } \\
\text { Laboratorios e Institutos de Salud Dr. } \\
\text { Carlos G. Malbrán) }\end{array}$ & http://sgc.anlis.gob.ar/ \\
\hline 37 & UMaza Digital, Universidad Maza. & http://repositorio.umaza.edu.ar/ \\
\hline 38 & $\begin{array}{l}\text { Biblioteca Digital UDA, Repositorio } \\
\text { Institucional de la Universidad de } \\
\text { Aconcagua. }\end{array}$ & http://bibliotecadigital.uda.edu.ar/ \\
\hline
\end{tabular}




\begin{tabular}{|c|l|l|}
\hline 39 & $\begin{array}{l}* * \text { El abrigo, Repositorio Institucional, } \\
\text { Servicio Meteorológico Nacional. }\end{array}$ & http://repositorio.smn.gob.ar/ \\
\hline 40 & $\begin{array}{l}\text { Repositorio Digital de la Universidad } \\
\text { Nacional de la Matanza. }\end{array}$ & $\begin{array}{l}\text { https://www.unlam.edu.ar/index.php?seccion=5\& } \\
\text { idArticulo=567 }\end{array}$ \\
\hline 41 & $\begin{array}{l}\text { Repositorio Institucional Unsam, } \\
\text { Universidad Nacional de San Martín. }\end{array}$ & https://ri.unsam.edu.ar/ \\
\hline 42 & $\begin{array}{l}\text { RiA UTN (Repositorio Institucional Abierto - } \\
\text { Universidad Tecnológica Nacional) }\end{array}$ & $\begin{array}{l}\text { https://www.utn.edu.ar/es/secretaria- } \\
\text { institucional-abierto-ria }\end{array}$ \\
\hline
\end{tabular}

** RI en el que las políticas se presentan explicitadas. 